\title{
Soziale Innovation zwischen Emanzipation und Anpassung
}

\begin{abstract}
Markus Pausch*
Zusammenfassung

Der Begriff „Innovation“ wurde lange vor seiner technologisch-ökonomischen Definition als auf die Zukunft gerichtetes soziales und emanzipatorisches Unterfangen verstanden, das in enger Verbindung zu den Werten der Aufklärung und der Entwicklung der modernen Demokratie steht. Im 20. Jahrhundert wurde er jedoch umdefiniert, vorrangig wirtschaftlich interpretiert und von der Ökonomie besetzt. Erst seit einigen Jahren hat sich eine neue Debatte über die soziale Dimension von Innovation unter dem Schlagwort "Soziale Innovation“ entwickelt, im Rahmen derer verschiedene Definitionen vorgeschlagen wurden. Die Begriffserwendung ist nichtsdestotrotz eher diffus geblieben und konzentriert sich auf Top-Down-Prozesse. Im vorliegenden Artikel sollen alternative Interpretationen beschrieben und diskutiert werden. Mit Hilfe einer Kategorisierung werden vier verschiedene Typen von Sozialer Innovation vorgeschlagen, die für zukünftige praktische Ansätze einen Rahmen bieten können.
\end{abstract}

Schlagwörter: Soziale Innovation, Emanzipation, Demokratie

\section{Social Innovation between emancipation and adaptation}

\section{Abstract}

Long before its technological and economic conceptualization, innovation was discussed as a social emancipatory process, closely linked to the emergence of modern democracy. The questioning of existing truths and hierarchies is integral part of the term innovation as is the creative creation of alternatives. In this article, the focus is on the different dimensions of social innovation. The diffuse use of the term shall be contrasted with a more categorized understanding of social innovation.

Keywords: Social innovation, emancipation, democracy

\footnotetext{
* Markus Pausch, Fachhochschule Salzburg. E-Mail: Markus.pausch@fh-salzburg.ac.at
} 


\section{Einleitung}

Gesellschaften unterliegen einem ständigen Wandel, der auf unterschiedliche Arten entstehen kann, etwa ungesteuert (oder zufällig) aus dem Zusammenwirken von einer Vielzahl von Faktoren, dialektisch durch die Synthese von widersprüchlichen Aspekten oder zielgerichtet und von konkreten AkteurInnen intendiert. Innovationen zählen in der Regel zum letzten dieser Fälle und können somit als eine Variante von Zukunftsgestaltung betrachtet werden, die sich auf unterschiedliche Zeithorizonte beziehen kann. Howald beschreibt Soziale Innovationen treffend als „intentionale Neukonfiguration sozialer Praktiken" (Howaldt 2014, 13). Wie diese Neukonfiguration jedoch entsteht, von wem sie intendiert wird und welche Ziele sie verfolgt, bleibt vorerst offen. Auch der räumliche und zeitliche Horizont kann variieren. ${ }^{1}$ In der aktuellen Begriffsverwendung entsteht häufig der Eindruck, als wäre Soziale Innovation lediglich eine technokratisch-planerische Lösung sozialer Probleme, die „von oben“, also von EntscheidungsträgerInnen oder BeamtInnen initiiert und durch Anwendung von Managementmethoden umgesetzt wird. Begriffe wie "Sozialmanagement" oder „Social Entrepreneurship“ sind eng mit diesem Verständnis Sozialer Innovation verknüpft. Auch die partizipativer gestalteten Open-Innovation- oder Governance-Prozesse sind als Top-Down-Verfahren angelegt. Demgegenüber wird in diesem Artikel aufgezeigt, dass es auch „von unten“, also aus der Zivilgesellschaft heraus initiierte Formen Sozialer Innovation gibt. Argumentiert wird, dass es in Hinblick auf Ziele, Methoden, AkteurInnen sowie räumliche und zeitliche Ausrichtung vier Typen von Sozialer Innovation gibt, die unterschiedliche historische Ursprünge und Bezugspunkte aufweisen. So geht das emanzipatorische Begriffsverständnis auf die Zeit der Aufklärung zurück und stellt Freiheit, Gleichheit und Solidarität in den Mittelpunkt. An diese normative Tradition schließen neuere Definitionen der OECD und der Europäischen Union an, die unter Sozialer Innovation eine Verbesserung der allgemeinen Lebensqualität verstehen. "Social innovation deals with improving the welfare of individuals and community through employment, consumption or participation, its expressed purpose being therefore to provide solutions for individual and community problems." (Website OECD) In diesem

$1 \mathrm{Zu}$ einer guten Übersicht über die bisherige Begriffsdebatte vgl. Moulaert et al. 2013
Verständnis ist auch eine enge Anbindung an demokratische Grundsätze enthalten. Die Demokratisierung von Staat und Gesellschaft gilt als wichtiges Ziel (vgl. Eschweiler/Hulgard 2012). Im Gegensatz zum emanzipatorischen Ansatz, der in der Regel von BürgerInnen oder der Zivilgesellschaft getragen ist, geht der Impuls hier jedoch von den politischen Institutionen aus. Das auf den Arbeiten des Ökonomen Joseph A. Schumpeter gründende Innovationsverständnis betrachtet die Veränderung sozialer Praktiken als Anpassung an die Folgen technologischer und ökonomischer Neuerungen bzw. deren Nutzung (vgl. Schumpeter 1926). Die jüngeren Ansätze aus der Soziologie, wie jene von Blumer (1975), Zapf (1989) oder Howaldt/Schwarz (2010), sehen das Ziel von Sozialen Innovationen in der Entwicklung von Lösungen für soziale Probleme. Im Gegensatz zum emanzipatorischen Zugang stellt dieser eine Problemlösung auf Basis von Governance ins Zentrum und trifft sich somit mit den Zugängen der OECD oder der EU. Der afro-kanadische Soziologe Yao Assogba (2010) beschreibt schließlich mit der lokal initiierten und anfangs subversiven Sozialen Innovation einen weiteren Typus, der sich durch seine lokale Begrenztheit und seine anfängliche Klandestinität auszeichnet.

Im vorliegenden Artikel werden diese Typen näher beschrieben und entlang der oben genannten Kategorien geordnet. Als Ausgangsbasis für die nähere Beschreibung wird Tabelle 1 verwendet. Zur genaueren Kategorisierung von Sozialer Innovation wird im Folgenden zwischen Zielen, Methoden, AkteurInnen sowie räumlicher und zeitlicher Ausrichtung unterschieden.

\section{Soziale Innovation als Emanzipation}

Der erste Typus Sozialer Innovation, der sich auf das Ziel der Emanzipation bezieht, hat historisch und begriffsgeschichtlich die längste Tradition. Die Idee, dass die Zukunft nicht von Gott gesteuert, sondern vom Menschen gestaltbar sei, hat sich in den Jahrzehnten der Aufklärung zeitgleich mit der Idee der modernen Demokratie entwickelt und mit der Französischen Revolution 1789 einen ersten konkreten Niederschlag gefunden. Die Vorstellung einer gestaltbaren Zukunft ist also historisch betrachtet ein relativ junges Phänomen und eine wichtige Voraussetzung für die Entstehung von Innovationen (Heintel 2009: 87).

Innovationen wurden damals nicht in erster Linie als Erfindungen erachtet, sondern als wesentliche gesellschaftliche Veränderungen, die im Einbezug 
Pausch: Soziale Innovation zwischen Emanzipation und Anpassung

\begin{tabular}{|c|c|c|c|c|}
\hline \multicolumn{5}{|c|}{ Tabelle 1: Typen von Sozialer Innovation } \\
\hline & $\begin{array}{l}\text { Typ 1: Soziale Innova- } \\
\text { tion als Emanzipation }\end{array}$ & $\begin{array}{l}\text { Typ 2: Soziale Innovation } \\
\text { als Anpassung an und Nut- } \\
\text { zung von Technologien }\end{array}$ & $\begin{array}{l}\text { Typ 3: Soziale Innova- } \\
\text { tion als Problemlösung }\end{array}$ & $\begin{array}{c}\text { Typ 4: Soziale Innova- } \\
\text { tion als lokale Normab- } \\
\text { weichung }\end{array}$ \\
\hline Ziele & Emanzipation & Gewinn/Profit & Planerische Problemlösung & $\begin{array}{l}\text { Lokale Problemlösung von } \\
\text { unten }\end{array}$ \\
\hline Methoden & $\begin{array}{l}\text { BOTTOM-UP durch Sozialre- } \\
\text { form oder Revolution }\end{array}$ & $\begin{array}{l}\text { TOP-DOWN durch Marketing } \\
\text { oder Open Innovation }\end{array}$ & $\begin{array}{l}\text { TOP-DOWN durch Govern- } \\
\text { ance }\end{array}$ & $\begin{array}{l}\text { BOTTOM-UP durch Subver- } \\
\text { sion und Teil-Öffentlichkeit }\end{array}$ \\
\hline $\begin{array}{l}\text { Initiatorlnnen/ } \\
\text { Akteurlnnen }\end{array}$ & $\begin{array}{l}\text { Revolutionäre oder } \\
\text { Reformer }\end{array}$ & UnternehmerInnen, ErfinderInnen & $\begin{array}{l}\text { Beamte oder Interessenver- } \\
\text { treterlnnen }\end{array}$ & Aktivistlnnen \\
\hline $\begin{array}{l}\text { Räumliche } \\
\text { Ausrichtung }\end{array}$ & Global, universell & Markt- und zielgruppenorientiert & $\begin{array}{l}\text { Staatlich, regional, konkrete } \\
\text { Organisation }\end{array}$ & Lokal \\
\hline $\begin{array}{l}\text { Zeitliche } \\
\text { Ausrichtung }\end{array}$ & Mittel- bis langfristig & $\begin{array}{l}\text { Kurz- und mittelfristig intendiert, } \\
\text { langfristig in der Auswirkung }\end{array}$ & Kurz- und mittelfristig & Mittelfristig \\
\hline
\end{tabular}

Quelle: Eigene Darstellung.

neuer Gruppen der Bevölkerung bzw. dem Hervorrufen von Veränderungen durch völlig andere AkteurInnen in der Gesellschaft lag. Sie galten somit auch als etwas, das die etablierte Ordnung bedrohte, und hatten daher im Mittelalter den Ruf von etwas Gefährlichem. Noch in der Renaissance betrachtete man Innovation in Kirchenkreisen gar als Häresie (Godin 2012: 8). Später wurde sie mit den Ideen der Französischen Revolution und jenen des Sozialismus in Verbindung gebracht (Godin 2012: 6). Im Begriffsverständnis dieser Zeit ist eine Soziale Innovation dann gegeben, wenn sie die Entlassung aus der väterlichen Gewalt, die Befreiung eines Sklaven oder insgesamt die Befreiung von Individuen aus Zwang, Unterdrückung und Ungleichheit zum Ziel hat, also eine - im Sinne der universalistischen Menschenrechte - alle Teile der Gesellschaft umfassende Emanzipation. Dieses Anliegen, das von Gründervätern der modernen Soziologie wie Auguste Comte geteilt wurde (vgl. Comte 1852), stieß auf breite Kritik unter konservativen Gesellschaftsschichten und Eliten. Die Arbeiten von William Lucas Sargant, einem englischen Ökonomen des späten 19. Jahrhunderts, zeigen dies auf. In einer kritischen Publikation mit dem Titel "The social innovators and their schemes" beschuldigt er die Sozialinnovateure seiner Zeit, schematisch und verschwörerisch mit subversiven und revolutionären Methoden zu arbeiten (Sargant 1858: iii-v). Anarchisten wie Pierre-Joseph Proudhon oder Sozialutopisten wie Robert Owen wurden um die Wende vom 19. ins 20. Jahrhundert der Gruppe dieser Sozialinnovatoren zugerechnet (Sargant 1860: 446).

Soziale Innovation galt zu jener Zeit also als revolutionäres und umstürzlerisches Programm zur Verwirklichung von Freiheit und Gleichheit. Diese revolutionäre Konnotation wurde später durch eine sozialreformerische ersetzt, wodurch der Innovationsbegriff auch auf herrschende Eliten weniger gefährlich wirkte (vgl. Godin 2015). In der Folge wurde er insgesamt seltener geführt, und dann meist weniger revolutionär als humanistisch. Damit bleibt auch der enge Zusammenhang zur Demokratie bestehen.

Soziale Innovation als Emanzipation teilt die Anliegen der modernen Demokratie, die als Methode zur Ermöglichung und Bewahrung gleicher Freiheit das Verwirklichungsprogramm emanzipatorischer Prinzipien darstellt, und zwar nicht nur als Staats- und Regierungsform, sondern überall dort, wo Ungleichheit und Unterdrückung bekämpft werden, also auch als Lebensform (vgl. Pausch 2017; Dewey 2008). Ziel Sozialer Innovation bleibt in diesem Entwicklungsstrang somit die Verbesserung der Lebensumstände der sozial Benachteiligten, deren Emanzipation sowie die Herstellung oder Bewahrung demokratischer Verhältnisse. Als Sonderform davon kann auch das bezeichnet werden, was unter dem Schlagwort „demokratische Innovationen " in den vergangenen Jahren in der Politikwissenschaft diskutiert wird, wie z.B. neue Formen der Beteiligung, Participatory Budgeting, BürgerInnen-Räte u.ä. (vgl. dazu Smith 2009). Demokratische Innovationen sind somit eine Unterform der als emanzipatorisch gedachten Sozialen Innovation. Auch ökologische Anliegen, wie die Bewahrung von Lebensraum indigener Völker oder der Erhalt von natürlichen Ressourcen etc., sind in den vergangenen Jahrzehnten als Themen des emanzipatorischen Zugangs hinzugekommen.

Das Ziel der Emanzipation wurde und wird von RevolutionärInnen und SozialreformerInnen, gegen- 
wärtig auch von AkteurInnen der Zivilgesellschaft, als Kampf für Freiheit, Gleichheit und Gerechtigkeit vertreten. Die AkteurInnen dieser Richtung sind global agierende NGOs wie Amnesty International, Greenpeace, aber auch losere Initiativen wie Occupy Wallstreet oder andere, oft globalisierungskritische Bewegungen, Vereine, BürgerInnen-Initiativen, aber auch Einzelpersonen wie die Friedensnobelpreisträger des Jahres 2014, Malala Yousafzai und Kaylash Satyarthi. Ihr Engagement gilt den unterdrückten, benachteiligten oder entrechteten Menschen sowie der Demokratisierung von Strukturen und Prozessen.

Methodisch ist eine große Bandbreite möglich, von Demonstrationen und zivilem Ungehorsam, der Organisation in NGOs oder in politischen Parteien über Vereine, Plattformen usw. Die Innovation an sich ist dann erfolgreich, wenn emanzipatorische Ziele erreicht sind. Als Beispiele sind die rechtliche Gleichstellung von Frauen ebenso zu nennen wie die Durchsetzung von Minderheitenrechten, die Verbesserung der materiellen Lebensbedingungen von armutsgefährdeten Menschen oder ökologische Nachhaltigkeit. Der zeitliche Horizont ist tendenziell mittel- bis langfristig angelegt. Die Maßnahmen sollen nachhaltig wirken und sich räumlich möglichst breit ausdehnen.

Historisch, aber auch gegenwärtig gibt es eine Vielzahl an emanzipatorischen Sozialen Innovationen, die häufig gegen den Willen der Regierenden vorangetrieben werden. Besonders hervorzuheben sind hierbei Frauenbewegungen, da diese bereits seit dem Beginn der Diskussionen über Soziale Innovation in der Aufklärung aktiv sind und dabei überwiegend pazifistisch agierten (vgl. de Gouges 1791). Als emanzipatorische Soziale Innovationen können auch die Aktionen von zeitgenössischen Frauenrechtlerinnen wie Femen oder Pussy Riot gesehen werden, die im öffentlichen Raum durch Kunstaktionen auf Unterdrückung und Ausbeutung von Frauen weltweit verweisen. Weniger provokativ, aber ebenso aktionistisch, treten andere Initiativen für die Rechte der Frauen ein, etwa Terre des Femmes, die als konkrete Soziale Innovation seit 2001 Aktionen durchführen, um ein Zeichen gegen Gewalt an Frauen zu setzen (vgl. Website Frauenrechte).

\section{Soziale Innovation als Anpassung und Nutzung (Closed und Open Innovation)}

Chronologisch deutlich nach der Herausbildung eines emanzipatorischen Zugangs zu Sozialer Innovation, nämlich erst zu Beginn des 20. Jahrhunderts und unter dem Eindruck der Industriellen Revolution der zweiten Hälfte des 19. Jahrhunderts, wurde der Innovationsbegriff 1911 von Joseph Schumpeter aufgegriffen (vgl. Schumpeter 1912) und auf die wirtschaftliche Entwicklung bezogen. Aus seiner einflussreichen ökonomischen Theorie heraus lässt sich ein zweiter Typus Sozialer Innovation ableiten, der als Anpassung an wirtschaftliche und technische Innovationen bzw. deren Nutzung interpretiert werden kann. Die Veränderung der sozialen Praktiken erfolgt damit nach Maßgabe dessen, was zuvor entwickelt wurde und sich auf dem Markt durchsetzen konnte. Dabei kommen nach jüngerer Interpretation zwei Wege in Frage: Closed Innovation oder Open Innovation.

Die so genannte Closed Innovation (geschlossene Innovation) entspricht dem Konzept von Joseph Schumpeter, demzufolge Innovationen spontan aus der Wirtschaft heraus entstehen und von engagierten UnternehmerInnen initiiert und durchgeführt werden. Sie zeichnen sich durch Kreativität und Risikobereitschaft aus und sind imstande, Neues zu denken und zu realisieren. Innovationen seien „die überragende Tatsache in der Wirtschaftsgeschichte der kapitalistischen Gesellschaft" (Schumpeter 2010: 93), durch die neue Güter und Berufe hervorgebracht und alte ausgemustert werden und die tiefgreifende gesellschaftliche Veränderungen nach sich ziehen. Innovationen sind bei Schumpeter Prozesse einer schöpferischen Zerstörung, die GewinnerInnen und VerliererInnen produzieren (Kurz 2005: 49).

In dieser knappen Zusammenfassung des Innovationsbegriffs nach Schumpeter stecken viele Implikationen, die für die weitere Begriffsdebatte der Sozialen Innovation von Bedeutung sind. Festzuhalten ist, dass Innovationen für Schumpeter vom Genius einzelner Personen abhängen, daher diskontinuierlich auftreten, aber viele NachahmerInnen finden. Im neueren, über Schumpeter hinausgehenden Verständnis von Closed Innovation dominiert der Gedanke, dass ein Unternehmen aus dem eigenen Wirken und durch interne, innovationsfördernde Prozesse, etwa durch systematische Forschung und Entwicklung, Neues in die Welt bringen kann (Chesbough 2006). Wesensmerkmal der Innovation bleibt bei Schumpeter wie bei der Closed Innovation das Neue, das sich dem Alten gegenüber als besser erweist und es daher meist verdrängt. Durch die Verdrängung des Alten gibt es konsequenterweise VerliererInnen von Innovationsprozessen. Diese Prozesse wirken sich tiefgreifend auf die Gesellschaft aus (vgl. Schumpeter 1926), denn die BürgerInnen bzw. Konsu- 
mentInnen müssen sich den Neuerungen aus Ökonomie und Technik anpassen. ${ }^{2}$ Die Soziale Innovation ist in diesem Begriffsverständnis lediglich die Folge- oder Nebenerscheinung technologisch-ökonomischer Innovationen. Ihr Ziel ist die Anpassung an neue Technologien und Produkte oder deren Nutzung.

Dieses Verständnis von Sozialer Innovation wurde in der Technikfolgenabschätzung und der Akzeptanzforschung weiter verfolgt und ausdifferenziert (Godin 2012: 39). Der Grund für die Akzeptanz von Neuem kann zum einen eben ein gewisser Anpassungsdruck sein. Wenn etwa Arbeitsgeräte durch neue Maschinen ersetzt werden, müssen die ArbeiterInnen diese Maschinen entweder anwenden oder den Verlust ihres Arbeitsplatzes riskieren. Zum anderen kann aber auch der Nutzen einer Innovation zur freiwilligen Nutzung derselben führen, weil dadurch ein Bedürfnis befriedigt oder Chancen ermöglicht werden, die im Übrigen auch für die breite Masse emanzipatorischen Charakter haben können. Dies ist der Fall, wenn der Kauf eines Autos zu höherer Mobilität beiträgt oder durch Kommunikationstechnologien der Informationszugang erhöht wird. Eine dritte Möglichkeit, um mit Innovation auf Akzeptanz zu stoßen, ist die Einbeziehung der künftigen KundInnen durch Open Innovation. Sie gilt als alternativer partizipativer Ansatz, in dem Akzeptanz durch Beteiligung entsteht (vgl. Faber 2008). Das Prinzip von Open Innovation ist die aktive strategische Nutzung möglichst breiten Wissens aus der Forschung, aber auch aus der Bevölkerung, von potenziellen KundInnen und innerhalb des eigenen Betriebs, um optimale Bedingungen für Innovationen zu ermöglichen (Chesbough 2006: 93 f.). Open Innovation greift auf unternehmensexterne Ideen und Ressourcen zurück und wird dadurch tendenziell partizipativ. Die AkteurInnen der Innovation sind dann nicht mehr nur ErfinderInnen, EntwicklerInnen oder ManagerInnen, sondern darüber hinaus auch KundInnen, ForscherInnen usw.

Besonders beachtenswert ist in diesem Zusammenhang, dass das Konzept von Open Innovation ganz bewusst auch von politischen AkteurInnen for-

2 Die Erforschung von Innovationsprozessen als auch die Demokratietheorie haben sich seit Schumpeters einflussreichen Definitionen rasant weiterentwickelt. So wird heute in der ökonomischen Innovationsforschung etwa die mystifizierte Rolle des kreativen Zerstörers stark relativiert und auf Struktur- und Kontext-Aspekte hingewiesen. ciert wird, ${ }^{3}$ und zwar nicht nur für die Entwicklung technischer oder ökonomischer Innovationen, die sich dann indirekt durch Anpassung und Nutzung auf Sozialpraktiken auswirken, sondern auch für die unmittelbare Entwicklung Sozialer Innovationen, die der hier vorgeschlagenen Kategorisierung zufolge eher in den Bereich der „Sozialen Innovation als Problemlösung" fallen und sich mit Governance-Prozessen überschneiden. Dabei setzt etwa die österreichische Bundesregierung ganz ausdrücklich darauf, dass die gezielte Öffnung von Innovationsprozessen nicht nur in Unternehmen, sondern auch im öffentlichen Bereich Anwendung findet, z. B. durch Crowdsourcing, User-Communitys u. ä. (vgl. Website Open Innovation).

In Hinblick auf die sozialen Nebeneffekte, die durch technische und ökonomische Innovationen und die Anpassung an diese erfolgen, sind auch emanzipatorische Entwicklungen möglich. So haben Technologien und Produkte in der Geschichte der Menschheit die Emanzipation gewisser Bevölkerungsgruppen erst ermöglicht (vgl. Königstorfer 2008). Die Druckerpresse von Gutenberg hat den Freiheits- und Gleichheitsgedanken ebenso vorangebracht wie jüngere Kommunikationstechnologien. Radio, Fernsehen, Internet und Smartphone haben den Zugang zu Information und politischer Mitsprache verbessert. Das Automobil und seine auf Maschinen basierende arbeitsteilige Produktion haben zur Entwicklung einer Mittelschicht beigetragen usw. Keineswegs also läuft die Soziale Innovation als Anpassung oder Nutzung dem Emanzipationsgedanken notwendigerweise entgegen. Er ist nur nicht ihr ausgesprochenes Ziel, und sie kann genauso gut das Gegenteil bewirken, denn im Vordergrund steht der ökonomische Nutzen.

Der räumliche und zeitliche Horizont der Innovation ist meistens klar definiert und tendenziell eher kurz- bis mittelfristig, auch wenn eine langfristige Etablierung am Markt das Ziel ist und die Veränderung der sozialen Praktiken in der Regel längere Zeit-

3 Im Übrigen gilt dies nicht nur für die Entwicklung technischer oder ökonomischer Innovationen, die sich dann indirekt durch Anpassung und Nutzung auf Sozialpraktiken auswirken, sondern auch für die unmittelbare Entwicklung Sozialer Innovationen, die der hier vorgeschlagenen Kategorisierung zufolge jedoch eher in den Bereich der „Sozialen Innovation als Problemlösung" fallen und sich mit Governance-Prozessen überschneiden. Die Vermischung der Innovationsfelder durch die Bundesregierung ist für eine klare Definition Sozialer Innovation wenig hilfreich. 
räume in Anspruch nimmt. Man konzentriert sich betriebswirtschaftlich auf Zielgruppen und arbeitet in der Regel mit klaren Zeitplänen. Betriebliche, aber auch öffentliche Innovationsentwicklung hat sich in den letzten Jahren und Jahrzehnten hoch professionalisiert und wird häufig von spezialisierten Abteilungen koordiniert.

\section{Soziale Innovation als Lösung sozialer Probleme (Governance)}

Nachdem der Innovationsbegriff über lange Zeit hinweg vorrangig ökonomisch geführt wurde, stieß er in den 1990er-Jahren auch im sozialen Bereich wieder auf Interesse. ${ }^{4}$ Verschiedene sozialwissenschaftliche und soziologische Interessenvertretungen und Netzwerke nahmen ihn in ihr Standardrepertoire an Themenfeldern auf. Soziale Innovation wurde nun nicht mehr lediglich als Anpassung oder Nutzung interpretiert, sondern neu definiert, ohne dass es aber zu einem einfachen Rückgriff auf die ältere emanzipatorische Verwendung gekommen wäre. In der deutschsprachigen Sozialwissenschaft ist die Debatte erst in den vergangenen zehn bis fünfzehn Jahren wieder angelaufen. Zwar hat Wolfgang Zapf bereits Ende der 1980er-Jahre darauf hingewiesen, dass Soziale Innovationen die Suche nach Lösungen für soziale Probleme zum Gegenstand haben, jedoch ergab sich daraus keine breit akzeptierte Begriffsverwendung und auch noch keine eigene Forschungslinie in den Sozialwissenschaften (Zapf 1989). Dies war erst in den 1990er-Jahren und um die Jahrtausendwende herum der Fall.

In einem Artikel von Gillwald aus dem Jahr 2000 heißt es: „Soziale Innovationen sind, kurzgefaßt,

4 So hat sich in den vergangenen Jahren etwa die Zahl an Publikationen, aber auch an Lehrgängen, Studien und Seminaren zum Thema "Innovation“ deutlich erhöht. Innovationsmanagement ist als neuer Zweig der Betriebswirtschaftslehre und anderer Subdisziplinen des Faches wie Innovation im Tourismus etc. stark auf dem Vormarsch. Im öffentlichen Sektor wird versucht, Innovation durch die Vergabe von Forschungsprojekten und Fördergeldern voranzutreiben. Und seit einigen Jahren hat sich im Zusammenhang mit sozialen Neuerungen der Begriff der „sozialen Innovation" wieder so stark etabliert, dass er auch von nationalstaatlichen Ministerien und europäischen Institutionen verwendet wird. Verschiedene sozialwissenschaftliche und soziologische Interessenvertretungen und Netzwerke haben diese Bezeichnung in ihr Standardrepertoire an Themenfeldern aufgenommen. gesellschaftlich folgenreiche, vom vorher gewohnten Schema abweichende Regelungen von Tätigkeiten und Vorgehensweisen. Sie sind überall in gesellschaftlichen Systemen möglich, im Ergebnis Verhaltensänderungen und verwandt, aber nicht gleich mit technischen Innovationen." (Gillwald 2000: 5) Diese Definition lässt viele Fragen offen, v.a. jene nach dem Unterschied zwischen einer Innovation und dem allgemeinen gesellschaftlichen Wandel. Außerdem werden innerhalb der Definition noch undefinierte oder noch näher zu definierende Begriffe wie „Regelungen“, „Tätigkeiten“ oder „Vorgehensweisen“ verwendet. Howaldt und Jacobsen unterstreichen, dass es bei sozialen Innovationen jedenfalls um die Suche nach Lösungen für soziale Probleme geht (Howaldt/Jacobsen 2010). Howaldt und Schwarz stellen fest, dass Soziale Innovationen eher als „deskriptive Metaphern im Kontext von Phänomenen des sozialen Wandels bzw. gesellschaftlicher Modernisierung“ Verwendung fänden: „Eine soziale Innovation ist eine von bestimmten AkteurInnen oder AkteurInnenKonstellationen intentionale zielgerichtete Neukombination bzw. Neukonfiguration sozialer Praktiken in bestimmten Handlungsfeldern bzw. sozialen Kontexten, mit dem Ziel, Probleme oder Bedürfnisse besser zu lösen bzw. zu befriedigen, als dies auf der Grundlage etablierter Praktiken möglich ist. Es handelt sich dann und insoweit um eine soziale Innovation, wenn sie - marktvermittelt oder ,non- bzw. without-profit - sozial akzeptiert wird und breit in die Gesellschaft bzw. bestimmte gesellschaftliche Teilbereiche diffundiert, dabei kontextabhängig transformiert und schließlich als neue soziale Praktiken institutionalisiert bzw. zur Routine wird." (Howaldt/Schwarz 2010: 54 f.) Aus dieser sehr komplex gehaltenen Definition ragen neben dem Kriterium des Neuen oder der Erneuerung vier entscheidende Aspekte hervor, nämlich

1. Intendierte Suche nach Lösungen für soziale Probleme,

2. Unabhängigkeit von ökonomischem Profit,

3. Breite gesellschaftliche Diffundierung und

4. Veränderung sozialer Praktiken.

Howaldt und Schwarz verweisen darauf, dass Innovationen nicht per se Gutes hervorbringen, sondern eben perspektivenabhängig sind und damit ambivalent (Howaldt/Schwarz 2010: 54). Sie entheben den Begriff der stark normativen Bedeutung, wie sie im emanzipatorischen Typus angelegt ist. Dass Soziale Innovationen im Unterschied zu technologi- 
schen oder ökonomischen nicht auf Profitsteigerung abzielen, ist weitgehend unumstritten. Versteht man mit Blumer ${ }^{5}$ die Entstehung sozialer Probleme als konstruktivistisch, so lässt sich die Innovation auf die Problemlösung hin zuspitzen. „Meine These ist es, dass soziale Probleme hauptsächlich Resultate eines Prozesses kollektiver Definition sind; sie existieren nicht unabhängig davon als eine Konstellation objektiver sozialer Bedingungen spezifischer Art.“ (Blumer 1975: 102) In seinem Fünf-Stufen-Modell verweist er sodann darauf, dass ein Problem zuerst von einer Gruppe als solches wahrgenommen, erkannt und definiert sowie danach zu öffentlicher Anerkennung gebracht werden müsse. An diesem Punkt kommt wiederum die Demokratie als Rahmenbedingung für Soziale Innovation ins Spiel, denn nur in demokratischen Gesellschaften besteht die Möglichkeit der Aushandlung von Problemen in der Öffentlichkeit.

Ab der dritten Stufe, der Mobilisierung von Handlungsstrategien, beginnt die Fokussierung auf die Lösung des sozialen Problems, die mit der Erstellung von Handlungsplänen und deren Umsetzung weitergeht (vgl. Blumer 1975). Folgt man diesem soziologischen Ansatz, so kann man auch die Soziale Innovation als neuartige Lösung eines sozialen Problems entlang der genannten fünf Stufen konzipieren - hier beginnt sie im Wesentlichen ab Stufe 3, der Mobilisierung von Handlungsstrategien, und konkretisiert sich in der folgenden Erstellung von Plänen und deren Umsetzung. Allen Definitionen von Innovation entsprechend, wäre noch die Akzeptanz und die Diffundierung hinzuzufügen, um den Kreislauf einer Sozialen Innovation abzuschließen. Dieser Zugang lässt sich weitgehend auch mit dem des österreichischen Soziologen Josef Hochgerner und seinem Modell der vier I vereinbaren. Er setzt den Beginn mit einer Idee an, der eine Intervention und eine Implementierung folgen und die einen gewissen Impact nach sich zieht (vgl. Hochgerner 2012).

Die AkteurInnen einer solcherart verstandenen Sozialen Innovation sind in der Regel VertreterInnen von Organisationen oder Institutionen, etwa Ministerien oder deren Unterabteilungen, die mit konkreten Aufgaben zur Lösung sozialer Probleme betraut sind und Sozialmanagement betreiben. Sie können aber

5 Blumer gilt nach wie vor als maßgebliche Quelle, wenn es um die Definition eines sozialen Problems geht. Das Verständnis von Sozialer Innovation als Problemlösung kann von seinem Fünf-Stufen-Modell eines sozialen Problems daher an Klarheit gewinnen. auch aus der Zivilgesellschaft kommen oder sich spontan aus den Lebenswelten heraus organisieren (im Sinne einer kritischen Öffentlichkeit). Da sie die Lösung eines sozialen Problems zum Ziel haben, müssen sie der Definition nach planerisch und koordiniert vorgehen, also auch einen gewissen Grad an Organisation aufweisen. Häufig kommen sie aus dem System heraus, also Topdown. Aber auch die Entstehung neuer Vereine oder Interessengruppen kann ein Teilaspekt der Sozialen Innovation sein. NGOs, die in der Regel sowohl auf die Lösung eines konkreten Problems abzielen, gehen weitgehend den Schritten von Blumer entsprechend vor, um ihre Ziele durchzusetzen. Die Legitimation erfolgt dabei durch die Akzeptanz der Zieldurchsetzung in der vertretenen Gruppe bzw. in Teilen der Bevölkerung. Dort, wo es sich um demokratische Rahmenbedingungen handelt, ist die Zieldurchsetzung in der Regel durch die Mehrheit der Bevölkerung legitimiert, oder - wie im Falle der Durchsetzung von Minderheitenrechten durch verfassungsmäßig vorgegebene Grundsätze.

Häufig wird diese Art der Sozialen Innovation, sofern sie aus Ministerien oder öffentlichen Stellen kommt, auch als demokratische Governance angelegt, also als eine Form von Entscheidungsfindung, die sich von streng hierarchischen GovernmentPrinzipien dadurch unterscheidet, dass sie möglichst viele Betroffene einzubinden versucht. Wie im vorigen Kapitel bereits kurz angedeutet, wird dafür auch die Bezeichnung der Open Innovation geführt. Unter beiden Schlagworten (Governance und Open Innovation) wird die Einbeziehung von Stakeholdern und Bevölkerung betont. Auf Ebene der Europäischen Union und mancher Mitgliedstaaten haben sich diese Governance-Prozesse in den vergangenen Jahren verstärkt. Mit den Prinzipien einer "good governance“ hat die EU bereits 2001 begonnen, sich dem Thema in der Gesetzgebung zu widmen. Die dazugehörigen Prinzipien lauten: Partizipation, Offenheit, Effektivität, Verantwortungsbewusstsein und Kohärenz. „Wie gut, sachgemäß und wirksam die Union ist, hängt davon ab, inwieweit die AkteurInnen in den Politikgestaltungsprozess - von der Konzipierung bis hin zur Durchführung - einbezogen werden. Verstärkte Teilhabe bewirkt größeres Vertrauen in das Endergebnis und die Politik der Institutionen." (Europäische Kommission 2001: 13) Die Beispiele für derartige Governance-Prozesse sind vielfältig. Sie reichen von Weißpapieren der EU über Leitbildprozesse oder Zukunftswerkstätten in Städten oder Regionen bis hin zu Demokratiereformen (vgl. www.partizipation.at). 
In der Realität laufen Governance-Prozesse oft über die Einbeziehung von organisierten InteressensGruppen wie NGOs ab. In der EU hat sich daraus u.a. der sogenannte zivile Dialog entwickelt (vgl. Pausch 2008: 136 f.). Auch auf nationalstaatlicher oder regionaler politischer Ebene findet man immer öfter Formen der Governance, etwa über Beiräte oder durch Konsultations-Verfahren. Diese organisierte und systematische Einbindung von zivilgesellschaftlichen AkteurInnen in Entscheidungsfindungsprozesse und in die Ausarbeitung von Innovationen zur Lösung von sozialen Problemen ist aus einer demokratiepolitischen Betrachtung heraus ambivalent. Es können dadurch zwar der Dialog gefördert und die Partizipation erhöht werden, aber es kann sich auch eine Alibi-Partizipation ergeben, die nur der Legitimation von politischen Entscheidungen dienen soll. Politische Verantwortungen können dadurch verschleiert werden, dass sich Ministerien oder Ämter auf die Einbeziehung von NGOs oder ExpertInnen ausreden. Governance-Methoden stoßen insbesondere dort an ihre Grenzen, wo starke Konfliktpositionen aufeinanderprallen und kein kleinster gemeinsamer Nenner gefunden werden kann. Für diese Fälle bleiben demokratische Abstimmungsverfahren in Parlamenten oder die Komptenzen von Ministerien und Ämtern unverzichtbar.

\section{Soziale Innovation als lokale Normabweichung}

Eine weitere Sicht auf Soziale Innovationen bietet schließlich der afro-kanadische Soziologe Yao Assogba mit Rückgriff auf verschiedene Klassiker der Soziologie wie Max Weber oder Raymond Boudon.

Laut Assogba ist eine Soziale Innovation die Antwort auf ein konkretes soziales und lokal begrenztes Problem, und zwar als Lösung einer als inakzeptabel oder unzufriedenstellend erlebten Situation. Meist liegt ein humanistisches Motiv zugrunde. Was nicht mehr länger akzeptabel scheint, soll geändert werden. In diesem Ausgangspunkt schwingt die vom französischen Philosophen Albert Camus beschriebene Notwendigkeit der Revolte gegen Unterdrückung, Unfreiheit und Ungerechtigkeit mit. Der Sklave, der seinem Herrn die Stirn bietet, steht am Beginn dieser Art der Sozialen Innovation, die er aber nicht nur für sich, sondern für alle fordert (vgl. Camus 1997; vgl. Pausch 2017).

Mit der Betonung des lokalen Aspekts unterscheidet sie sich von den zuvor genannten Annahmen, auch wenn das Ziel im Grunde hier wie dort in der Problemlösung besteht. Während die Initiative beim zuvor besprochenen Typus jedoch von der Regierung oder der Administration ausgeht, wird hier der Veränderungsbedarf von unten nach oben getragen, da er von den Regierenden nicht gesehen oder bewusst ignoriert wird. Der Ansatz hat somit im Gegensatz zu Governance-Prozessen oder Open Innovation, die jeweils von den Eliten initiiert werden, den Ausgangspunkt im Kleinen. Nicht dort, wo die institutionalisierte Macht und ihre AkteurInnen ansetzen, beginnt diese Art der Innovation, sondern im Gegenteil dort, wo diese institutionalisierte Macht „von unten“ herausgefordert oder einfach übergangen wird.

Die Soziale Innovation entwickelt sich hier also „wenig kodifiziert“, in einer „certaine clandestinité, also potenziell im Untergrund, da es sich um abweichlerische Lösungen handelt, die einen Verstoß gegen etablierte Regeln implizieren. Merkmal einer Sozialen Innovation ist hier zudem das Abzielen auf eine soziale Finalität und dahinterliegende Werte sowie - neben Diffundierung und Akzeptanz - die Institutionalisierung als letzte Etappe, die wiederum einer staatlichen Intervention bedarf (Assogba 2010: 2). „Die Innovation schreibt sich somit in eine Dialektik ein, die einerseits den Bruch mit der Institution, andererseits die Neukonstruktion der Institution als neuer Norm beinhaltet, die ihrerseits wiederum infrage gestellt werden kann.“ (Assogba 2010: 2) ${ }^{6}$ Während also bei der Sozialen Innovation als Problemlösung ein koordinierter und geplanter Akt der Governance durch legitimierte und organisierte AkteurInnen gemeint ist, weist Assogba auf das subversive Element von Innovationen hin. Es geht um einen Prozess von unten nach oben. Das Streben nach sozialer Finalität in einem lokal begrenzten Raum zeigt die Normativität der Innovation auf. Nicht Management oder Governance, sondern die Gestaltung und Veränderung des Sozialen stehen im Vordergrund. Die AkteurInnen dieser Art von Innovation agieren zumindest anfangs oft im Unter-, zumindest aber im Hintergrund (also subversiv), denn ihre Lösungen können für das Establishment (also die Norm setzenden AkteurInnen) bedrohlich sein. Die Methode ist schließlich dennoch auf die Einbeziehung verschiedener AkteurInnen angewiesen, um Akzeptanz und demokratische Legitimität zu erreichen, kann aber mit

6 Eigene Übersetzung ins Deutsche. Im Original heißt es: "L'innovation s'inscrit donc dans une dialectique incluant, d'une part, une rupture avec l'institution mais, d'autre part, une construction de l'institution en devenant la nouvelle norme qui sera à son tour éventuellement défiée." (Assogba 2010: 2) 
einem Stufenplan verglichen werden, der im Geheimen beginnt und sich nach und nach Öffentlichkeit verschafft. Das bereits erwähnte Modell von Blumer ist somit auch bei diesem Typus relevant.

Als die Bestandteile einer Sozialen Innovation nennt Assogba folgerichtig:

1. Ausgangspunkt ist ein konkretes lokales Problem, auf das mit einer neuartigen Lösung reagiert wird.

2. Ziel ist nicht nur die Lösung des konkreten Problems vor Ort, sondern eine soziale Finalität, die auf Werten wie Gleichheit oder Gerechtigkeit beruht.

3. Getragen wird die Soziale Innovation von einer Vielzahl an AkteurInnen. Sie geht von einigen wenigen lokal Handelnden aus und verbreitet sich über einen demokratischen Prozess und Verhandlungen sowie Netzwerke.

4. Durch Verbreitung (Diffusion) und Adaption der Sozialen Innovation außerhalb ihres ursprünglichen, lokalen Anwendungsrahmens beweist sie Effizienz. Sie gilt als erfolgreiches Experiment, das auf ähnliche Situationen übertragbar ist.

5. Die Institutionalisierung steht am Ende als letzte Etappe einer erfolgreichen Sozialen Innovation. Dies ist meist mit einer staatlichen Intervention verbunden. (Assogba 2010: 2)

Assogba geht noch einen Schritt weiter und unterscheidet in Anlehnung an ein Modell von Mendras und Forsé (1983) zwischen verschiedenen Akteurstypen, die im Prozess der Sozialen Innovation nacheinander auftreten. Am Beginn dieses Prozesses stehen die verdeckt agierenden InitiatorInnen. Sie überzeugen in einem nächsten Schritt einige wenige PionierInnen, die bereit sind, die Innovation umzusetzen und mitzutragen. Es folgen jene, die überzeugt werden können und die eine wichtige Rolle als anerkannte Trendsetter im lokalen Raum spielen. Durch ihre Vorbildwirkung schließen sich weitere an, die zwar eher skeptisch sind, aber nachahmen, ohne überzeugt zu sein. Die als TraditionalistInnen bezeichnete letzte Gruppe ist am schwersten zu einer Änderung ihrer sozialen Praktiken zu bringen, folgt schließlich im Falle einer erfolgreichen Innovation aber doch. Assogba nennt die demokratische Willensbildung, den Austausch zwischen verschiedenen AkteurInnen, als wesentliches Kriterium für die von ihm ins Auge gefassten lokal begrenzten Innovationen. Als Beispiele nennt er die Organisation Le Dépanneur Sylvestre, eine spontan entstandene Initiative zur Unterstützung sozialer Inklusion, die obdachlose und bedürftige Menschen u.a. mit Nahrung versorgt. Das deutsche Pendant dazu wäre die Organisation Tafel, die
1993 in Berlin als lokale Initiative gegründet wurde und sich seither rasant in Europa ausgebreitet hat. Auch die Gründung von Cap Anamur, einer Rettungsaktion für Bootsflüchtlinge, oder Orte wie das italienische Dorf Riace, wo bewusst MigrantInnen angesiedelt wurden, um der Abwanderung und Alterung entgegenzuwirken, entsprechen der lokalen Sozialen Innovation, die Assogba beschreibt. Als jüngste Beispiele können darüber hinaus die vielen zivilgesellschaftlichen Aktionen zur Beherbergung oder Integration von Zuwanderern begriffen werden, die 2015 nach Europa kamen. In all diesen Fällen haben AkteurInnen der Zivilgesellschaft von der Norm abweichende Lösungen für konkrete lokale Probleme gefunden.

Auffallend dabei ist, dass in manchen Fällen dieser Sozialen Innovation als lokaler Normabweichung die Zivilgesellschaft gegen institutionalisierte Macht aufbegehrt, in manchen Fällen aber auch humanitäre Hilfeleistungen gestellt werden, weil die institutionalisierte Macht versagt oder ihrer Aufgabe nicht auf zufriedenstellende Weise nachkommt. So wurde in den Jahren 2015 und 2016 vom Staat Östereich ein Teil seiner Aufgaben in der Asylpolitik auf privatwirtschaftliche Träger ausgelagert. Man kann dies als eine Soziale Innovation im Sinne des dritten beschriebenen Typus (Lösung sozialer Probleme) interpretieren. Neben dieser und zum Teil gegen diese Art der Problemlösung hat sich eine lokale Normabweichung humanitären Antriebs formiert, Flüchtlingshilfe organisiert und damit den Staat einerseits herausgefordert und andererseits entlastet. Passend zu den Annahmen Assogbas sind die Aktivitäten der FlüchtlingshelferInnen bzw. der entsprechenden NGOs nicht darauf beschränkt, das unmittelbare lokale Problem zu lösen. Vielmehr wird auf eine gesamtgesellschaftliche Lösung abgezielt, die auf humanistischen Werten beruht und der zur Norm verholfen werden soll.

\section{Ausblick}

„Soziale Innovation“ wurde als eine Form von Zukunftsgestaltung zu unterschiedlichen Zeiten mit unterschiedlichen Relevanzgesichtspunkten diskutiert: als emanzipatorisches Projekt im Anschluss an die Aufklärung; als Anpassung an und Nutzung von technologischen und ökonomischen Neuerungen im Zuge der Marktwirtschaft; als soziale Problemlösung und Governance durch organisierte AkteurInnen; und als subversive Normabweichung und lokal initiierte Zukunftsgestaltung von unten. Alle vier Ausprägungen 
sind nach wie vor relevant und kommen im Diskurs sowie im Selbstverständnis verschiedener AkteurInnen vor. Teilweise überschneiden sich die Ziele und Methoden der vier genannten Arten, teils grenzen sie sich voneinander ab. Dabei fällt auf, dass auch jene Innovationsmodelle, die von wirtschaftlichen oder politischen EntscheidungsträgerInnen Top-down initiiert werden, zunehmend dazu übergehen, die breitere Einbeziehung der Bevölkerung zu forcieren. Die Schlagworte dazu heißen Open Innovation und Good Governance. Auch das Verhältnis zwischen lokaler Orientierung und globalem Anspruch hat sich gewandelt. Während die emanzipatorische Bewegung häufig universalistische und globale Missstände zum Ausgang nimmt und so über große Systemveränderungen ins Lokale hineinzuwirken versucht, ist der Weg bei lokalen Initiativen der umgekehrte. Sie starten im kleinen lokalen Raum und dehnen sich zwar nicht notwendigerweise, aber doch potenziell auf größere Räume aus. Die Unterscheidung der vier genannten Typen könnte im besten Fall dazu beitragen, die Debatte über Soziale Innovationen in Hinblick auf Ziele, Methoden, AkteurInnen und räumliche Ausrichtung zu vertiefen, ohne dabei normative Setzungen vorzunehmen oder nur eine einzige Sicht gelten zu lassen.

\section{Literatur}

Assogba, Y. (2010) : Théorie Systémique de L’Action Sociale et Innovation Sociale. Série Recherches, numéro 31, 1-16.

Blumer, H. (1975): Soziale Probleme als kollektives Verhalten. In: Hondrich, K. O.: Menschliche Bedürfnisse und soziale Steuerung. Reinbek: Rowohlt, 102-113.

Camus, A. (1997): Der Mensch in der Revolte. Reinbek: Rowohlt.

Chesbough, H. W. (2006): Open Innovation. The New Imperative for Creating and Profiting from Technology. Boston Massachusetts: Harvard Business School Press.

Comte, A. (1852): Catéchisme positiviste ou Sommaire Exposition de la Religion Universelle en onze entretiens systématiques entre une femme et un prêtre de l'Humanité. Un document produit en version numérique par Jean-Marie Tremblay, édition electronique 2002.

De Gouges, O. (1791): Déclaration des Droits de la Femme et de la Citoyenne. Online: https://rownosc.info/media/ uploads/deklaracja_praw_kobiety_i_obywatelki.pdf; [27.08.2017].

Dewey, J. (2008): The later works, Vol. 11: 1935-1937, hrsg. von Boydston, Jo Ann. Carbondale: Southern Illinois University Press.
Eschweiler, J./Hulgard, L. (2012): 'Social Innovation and Deliberative Democracy'. WP, 12/o4, EMES European Research Network.

Europäische Kommission (2001): Weißbuch: Europäisches Regieren. Brüssel: KOM (2001) 428.

Faber, M. J. (2008): Open Innovation. Ansätze, Strategien und Geschäftsmodelle. Wiesbaden: Gabler Edition Wissenschaft.

Gillwald, K. (200o): Konzepte sozialer Innovation. Working Paper, Wissenschaftszentrum Berlin für Sozialforschung, Berlin.

Godin, B. (2012): Social Innovation: Utopias of Innovation. Working Paper No 11, Project on the Intellectual History of Innovation, Quebec.

Godin, B. (2015): Innovation Contested. The idea of innovation over the Centuries. London: Taylor and Francis.

Heintel, P. (2009): Zukunftsgestaltung. Ein philosophischer Essay. In: Popp, R./Schüll, E. (Hg.): Zukunftsforschung und Zukunftsgestaltung. Beiträge aus Wissenschaft und Praxis. Berlin, Heidelberg: Springer, 87-98.

Hochgerner, J. (2012): Social Innovation: The Concept and its Potential. Conference Paper Kiev.

Howaldt, J./Jacobsen, H. (Hg.) (2010): Soziale Innovation. Auf dem Weg zu einem postindustriellen Innovationsparadig$m a$. Wiesbaden: VS Verlag für Sozialwissenschaften.

Howaldt, J./Schwarz, M. (2010): „Soziale Innovation“ im Fokus. Skizze eines gesellschaftstheoretisch inspirierten Forschungskonzepts. Bielefeld: Transcript Verlag.

Königstorfer, J. (2008): Akzeptanz von technologischen Innovationen. Nutzungsentscheidungen von Konsumenten dargestellt am Beispiel von mobilen Internetdiensten. Wiesbaden: Springer.

Kurz, H. D. (2005): Joseph A. Schumpeter. Ein Sozialökonom zwischen Marx und Walras. München: Metropolis Verlag.

Mendras, H./Fors, M. (1983) : Le changement social, tendances et paradigmes. Paris : Armand Colin.

Moulaert, F./MacCallum, D./Mehmood, A./Hamdouch, A. (Hg.) (2013): The International Handbook of Social Innovation. Collective Action, Social Learning and Transdisciplinary Research. Northampton: Edward Elgar Publishing.

Pausch, M. (2008): Europas vergessene Öffentlichkeit. Probleme einer Demokratisierung der Europäischen Union. Frankfurt am Main: Peter Lang Verlag.

Pausch, M. (2017): Demokratie als Revolte. Zwischen Alltagsdiktatur und Globalisierung. Baden-Baden: Nomos.

Sargant, W. L. (2009/1858): Social Innovators and their Schemes. Cambridge: Cambridge Scholars Publishing.

Sargant, W. L. (1860): Richard Owen and His Social Philosophy. London: Smith, Elder \& Co.

Schumpeter, J. A. (1926/1911): Theorie der wirtschaftlichen Entwicklung. München/Leipzig: Duncker \& Humboldt.

Schumpeter, J. A. (1950/1942): Kapitalismus, Sozialismus, Demokratie. Bern: Francke. 
Schumpeter, J. A. (2010/1939): Konjunkturzyklen. Eine theoretische, historische und statistische Analyse des kapitalistischen Prozesses. Stuttgart: UTB.

Smith, G. (2009): Democratic Innovations. Designing Institutions for Citizen Participation. Cambridge: Cambridge University Press.

Website Frauenrechte: http://www.frauenrechte.de/online/index.php/themen-und-aktionen/fahnenaktion, [6.07.2017].

Website OECD: http://www.oecd.org/cfe/leed/Forum-Social-Innovations.htm [09.11.2016].

Website Open Innovation: http://openinnovation.gv.at/, [26.09.2017]

Zapf, W. (1989): Über soziale Innovationen. Soziale Welt, 40 $(1-2), 170-183$. 